

\title{
PERMEATION MEASUREMENTS ON IN SITU GROWN CERAMIC MFI TYPE FILMS
}

\author{
E.R. Geus ${ }^{\star}$, W.J.W. Bakker ${ }^{\star \star}$, P.J.T. Verheijen ${ }^{\star *}$, M.J. den Exter", J.A. Moulijn ${ }^{\star \star}$, H. van Bekkum ${ }^{\star}$ \\ *Delft University of Technology, Department of Applied Chemistry, Julianalaan 136, 2628 BL Delft, \\ Netherlands \\ ** Delft University of Technology, Department of Chemical Technology, Julianalaan 136, 2628 BL Delft, \\ Netherlands
}

\section{ABSTRACT}

Continuous MFI type films were grown on macroporous ceramic clay-type supports. Permeation experiments were performed using a Wicke-Kallenbach experimental set-up. Applying helium as an inert carrier gas, the permeation behaviour of strongly (n-butane, isobutane), and weakly adsorbing species (neon, argon, methane) was studied at room temperature for both 'pure' gases, and binary mixtures. Significant differences in permeation behaviour were found between strongly and weakly adsorbing gases, although steady state permeation rates varied less than one order of magnitude. The lower diffusivity of heavier alkanes is compensated by the higher sorbate concentration within the zeolite micropores. Accordingly, only low selectivities were found for binary mixtures, where the permeation rate is governed by the slowest moving species. For sorption near or within the Henry region, substantially higher selectivities were obtained. The presence of a macroporous layer on one side of the membrane will lead to reduced permeation rates, especially for strongly adsorbing molecules.

\section{INTRODUCTION}

During the last decade much attention has been paid to the preparation of ceramic membranes with molecular sieve characteristics for separation and catalysis. Notwithstanding numerous efforts, the preparation of crystalline molecular sieve membranes, or generally ceramic zeolite membranes, proved hard to accomplish. Some work has been devoted to the permeation through one or several zeolite crystals, embedded in epoxy resin [1-3]. Recently, the permeation through unsupported zeolite (MFI type) films was reported [4]. Here we report the preparation of MFI type films on a clay support.

Several publications have been forwarded by Barrer [5-7] to describe molecular flow through zeolite membranes. Permeation studies on pure zeolite membranes may shed light on the still existing differences in diffusivity between uptake measurements and PFG NMR self diffusivity data [8].

A seemingly underestimated aspect of diffusion within and through zeolites involves the modelling of adsorption. For reasons of convenience and simplicity the Langmuir isotherm is commonly used as the adsorption model, notwithstanding the fact that several prerequisites (e.g. heat of adsorption irrespective of sorbate concentration) are not met, especially at high sorbate concentrations. As most diffusion studies are limited to the Henry region, zeolite diffusion near saturation conditions and in the liquid phase are poorly studied. Concurrently, adsorption studies are generally performed well below 1 bar, for which application of the Langmuir isotherm does not lead to substantial error. Most membrane processes will however be run at high sorbate concentrations as high feed pressures are generally applied, whereas high permeate pressures are favourable from an engineering point of view. It is therefore the purpose of this study to discuss the diffusion process through zeolite membranes also for adsorption near saturation. 


\section{THEORY}

For steady-state permeation, the flow rate within the zeolite film is constant. Assuming both adsorption to and desorption from the zeolite framework to be relatively fast processes as compared to intracrystalline diffusion, permeation is fully governed by mass transfer through the zeolite micropores:

$$
\phi_{\text {mol }}=-A_{m} \cdot D(q(x)) \cdot \frac{\partial q(x)}{\partial x}
$$

with $A_{m}$ the membrane surface area $\left(\mathrm{m}^{2}\right), D(q(x))$ the concentration dependent Fickian diffusivity $\left(\mathrm{m}^{2} / \mathrm{s}\right)$, $q$ the sorbate concentration $\left(\mathrm{mol} / \mathrm{m}^{3}\right)$, and $x$ the distance from the feed side $(\mathrm{m})$.

Diffusion as a result of a total Gibbs free energy gradient may be described by Fickian diffusion, driven by a concentration gradient, according to Darken [9]:

$$
D^{F i c k}(q)=D^{s e l f}(q) \cdot \frac{\partial \ln p}{\partial \ln q}
$$

In order to develop a complete model for intracrystalline diffusion, the sorbate concentration dependence of both $D^{\text {self }}$ and the Darken factor has to be taken into account. The Langmuir adsorption isotherm is generally used, although several other adsorption isotherms have been proposed. Applying Langmuir's isotherm it was argued by Barrer [7] that the decrease of $D^{\text {self }}$ will be proportional to the sorbate concentration. Thus a constant apparent diffusivity is expected since the decrease in $D^{\text {self }}$ is fully compensated by the Darken correction. From PFG NMR data it was established that $D^{\text {self }}$ is not linearly decreasing with the sorbate concentration, and for high sorbate concentrations the decrease in $D^{\text {self }}$ is overruled by the Darken factor [10]. This is further supported by experimental work of Caro et al. [11] on the self diffusivity of methane, ethane, and propane in silicalite. They proposed a modified free volume theory, relating the molecular mean jump length $\left(\left\langle\left.\right|^{2}\right\rangle\right)$ to $D^{\text {self: }}$

$$
D^{s \theta l f}=\frac{\left\langle I^{2}\right\rangle}{6 \cdot \tau}=\text { Const } 1 \cdot v_{f}^{2 / 3} \cdot \Gamma\left(\frac{5}{3}, \frac{\text { Const2 }}{v_{f}}\right)
$$

in which $\tau$ is the mean residence time between two succeeding jumps, $v_{f}$ the mean free volume, and $\Gamma$ denotes the incomplete gamma function. The above dependency has however unfavourable properties for near zero sorbate concentrations. A simple but adequate description proved to be:

$$
D^{s \theta 1 f}=D_{0} \cdot \exp \left(-\frac{\gamma \cdot q}{q_{c}-q}\right)
$$

in which $D_{0}$ denotes the intrinsic self diffusivity, and $\gamma$ and $q_{c}$ are fit parameters, related to the model in equation (3). This model approximates equation (3) for large values of the sorbate concentration, and has a linear dependence at low concentrations.

The theoretical flow through the membrane may then be calculated from:

$$
\int_{0}^{l_{m}} \phi_{m o I} \cdot d x=-A_{m} \int_{q_{T}}^{q_{p}} D^{s e I f}(q) \cdot \frac{d \ln p}{d \ln q} \cdot d q
$$

with $I_{m}$ the membrane thickness $(m)$, and $q_{F}, q_{p}$ the sorbate concentration on feed and permeate side, 
respectively.

The concentration profile over the zeolite film can be calculated from equation (5) as well. Within the Henry region it will suffice to assume a constant diffusivity, so a linear concentration profile is calculated. For strongly adsorbing molecules like $n$-butane or isobutane, sorption on the feed side will generally occur out of the Henry region, even at relatively low (partial) pressures. Therefore, the diffusivity will vary over the zeolite film and the permeate flow is not expected to be directly proportional to the imposed concentration or pressure difference over the membrane.

\section{EXPERIMENTAL}

Clay supports were prepared according to [12], the basic material being a standard clay suspension for the preparation of China (Koninklijke Porceleyne Fles; Delft, Netherlands). After calcination at $900^{\circ} \mathrm{C}$, the supports were polished to smooth disks (diameter $23 \mathrm{~mm}$; thickness $1.5 \mathrm{~mm}$ ) on the same clay material to avoid contamination. A synthesis mixture for silicalite-1 (Si-rich MFI) according to [13] was prepared by mixing silica (aerosil 200, Degussa), sodium hydroxide (Baker), and tetrapropylammonium bromide (TPABr; CFZ, Zaltbommel, Netherlands) in water. The mixture was aged for 1-6 hours at room temperature, and put into a $30 \mathrm{ml}$ teflon lined autoclave with a clay support disk. Crystallization times were varied between $1-5$ days at $180^{\circ} \mathrm{C}$. The resulting MFI/clay composites were washed with water and ethanol and dried at $50^{\circ} \mathrm{C}$ in air. Activation of the zeolite phase (removal of the template ions) was performed by calcination (> 10 hours) in air at $400^{\circ} \mathrm{C}$ (heating rate $1^{\circ} \mathrm{C} / \mathrm{min}$ ).

As-synthesized and calcined $\mathrm{MFl}$ /clay composites were attached to a thick perforated stainless steel disk with a gas tight epoxy resin (Araldit; Ciba-Geigy) leaving a well-defined free membrane surface area of approximately $1.5 \mathrm{~cm}^{2}$ open. The membrane was installed in a cell, the zeolite film facing the feed side. Permeation experiments (steady state and transient state) were performed at room temperature using a Wicke-Kallenbach set-up (negligible pressure difference over the membrane) with helium as an inert carrier gas. The feed composition $(0.1-100 \%)$ was controlled by mass flow controllers, while analysis of the permeate was carried out by mass spectrometry. High purity gases (helium, neon, argon, methane (>99.99\%), n-butane, isobutane (99.95\%); Intomar, Netherlands) were used. Calibration of the mass spectrometer was carried out daily using calibration gas mixtures in helium (appr. 1000 $\mathrm{ppm})$. Partial pressures on the permeate side did not exceed $100 \mathrm{~Pa}$.

\section{RESULTS AND DISCUSSION}

In Figure 1a and b Scanning Electron Microscope pictures of the prepared MFI/clay composite films are shown. Only the support top and side surfaces are fully covered with essentially a monolayer of intergrown, randomly oriented MFI type crystals with a film thickness of 50-80 $\mu \mathrm{m}$. On the bottom side that is facing the teflon lining during crystallization, some separate analcime crystals are formed. Analcime crystallizes when the Si/Al ratio is out of the synthesis region for MFI. It seems that upon hydrothermal treatment the synthesis mixture between the support and the teflon lining is enriched in aluminium by leaching from the clay phase. As-synthesized MFI/clay composite films proved to be gas tight for all gases up to $100 \%$ of feed gas. 




Figure 1a. Top view of the in situ grown MFI/clay composite



$50 \mu \mathrm{m}$

Figure $1 \mathrm{~b}$. Cross section of the MFI/clay composite

Some typical transient state permeation results for 'single' components (15\% in helium, being 0.24 bar partial pressure) at room temperature are shown in Figure 2. Under these conditions the steady state permeation rates are $6.0^{*} 10^{-8}$ (methane), $4.0^{\star} 10^{-8}$ (neon), $2.9^{*} 10^{-8}$ ( $\mathrm{n}$-butane), $1.3^{*} 10^{-8}$ (isobutane) $\mathrm{mol} / \mathrm{s}$. Weakly adsorbing gases (methane, neon) reach steady state permeation rates within 7-9 minutes, whereas heavier molecules are only detected after 4-5 minutes and reach steady state after one hour on stream. Steady state permeation of $n$-butane is twice as fast as isobutane, which is attributed to the lower diffusivity of isobutane. This has been observed previously for a one crystal silicalite-1 membrane [1a]. 


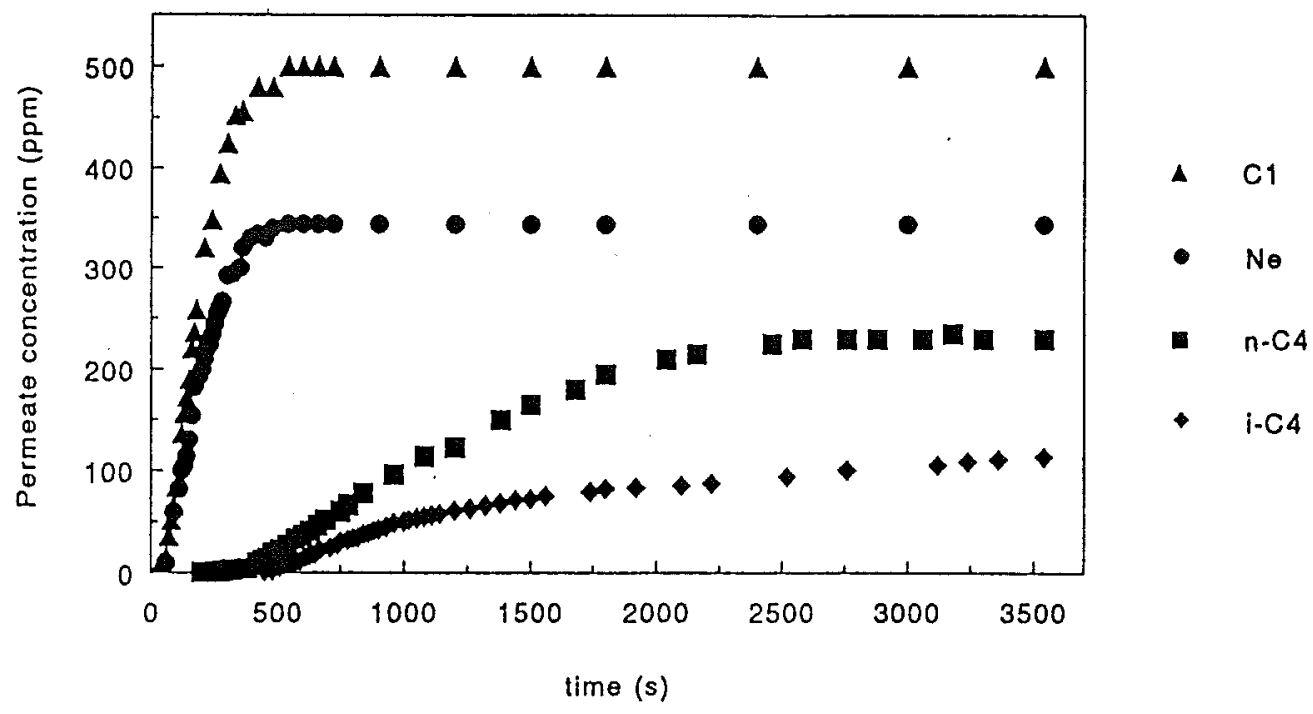

Figure 2. Transient state permeation at room temperature for 'pure' gases (feed: $15 \%$ in helium)

In Figure 3 the permeation behaviour of an equimolar binary mixture of isobutane and neon (15\%/15\% in helium) is shown. Permeation of neon starts of similar to the pure gas measurement, but deviates after 2-3 minutes to reach a temporary maximum. Thereafter the neon flow decreases to reach a steady state permeation rate that is substantially lower than the 'pure' gas value. The presence of neon had no noticeable effect on the isobutane permeation. At steady state the observed separation factor is only 1.3 (nearly non selective), whereas the ideal separation factor (based on pure gas permeabilities) under similar conditions is equal to 3 . It is concluded that isobutane, being far more abundant in the zeolite framework, reduces the neon permeation rate. This may be envisaged as neon molecules being trapped within a continuous stream of isobutane molecules. The reduced mobility of fast moving molecules in zeolite micropores due to the presence of slowly moving species has been reported previously for methane in silicalite with preadsorbed benzene molecules [14]. The self diffusion of methane was found to be reduced over two orders of magnitude.



Figure 3. Transient state permeation of a $(15 \% / 15 \%)$ mixture of neon and isobutane in helium at room temperature, and the pure gas/helium permeation for comparison. 
As the isobutane feed concentration is decreased, while the neon concentration is maintained at 0.24 bar, the selectivity remains constant down to a $3000 \mathrm{~Pa}$ isobutane feed (Figure 4). Only a minor increase in neon permeability (40\%) was observed, in accordance with the still near to saturation inner zeolite concentration of isobutane. The permeation rate for isobutane deviates slightly from being proportional to the feed concentration, and remained non-detectable $\left(<5.10^{-13} \mathrm{~mol} / \mathrm{s}\right)$ for very low feed pressures $(160 \mathrm{~Pa})$, even after 1 hour on stream. At the same low partial pressure the flow rate for neon and argon amounted $5.10^{-11} \mathrm{~mol} / \mathrm{s}$, whereas $\mathrm{n}$-butane was again non-detectable. Thus, an ideal separation factor of at least 100 was found.

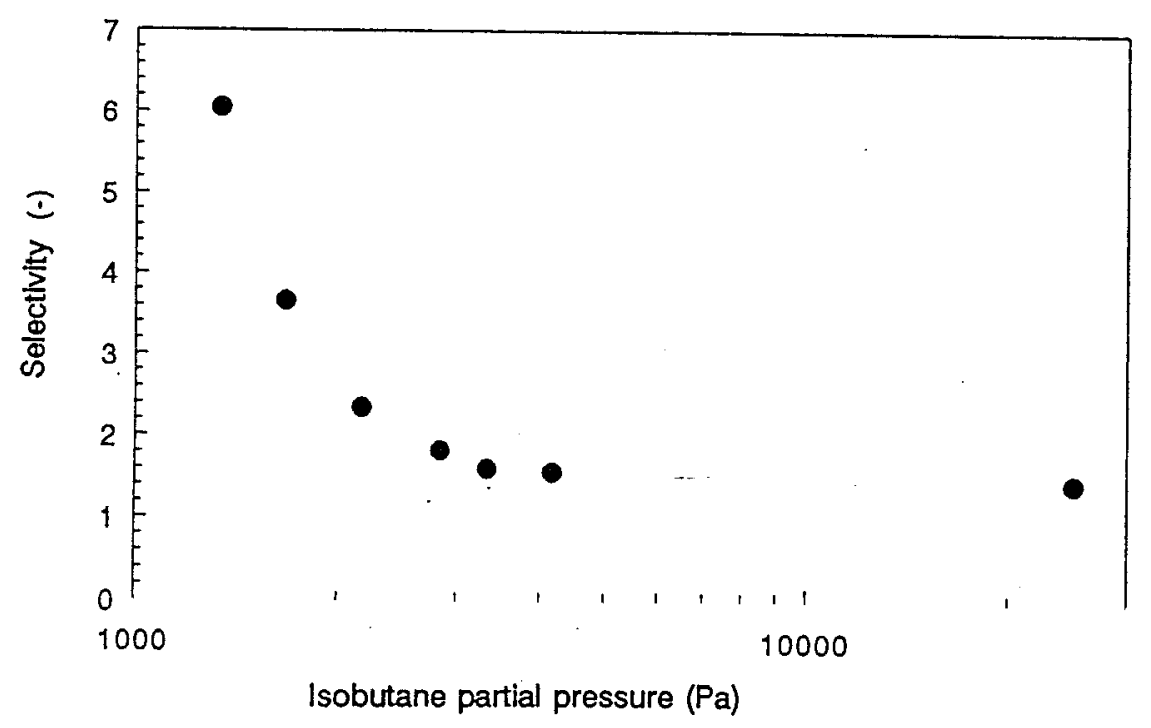

Figure 4. Neon/isobutane selectivity $\left((\mathrm{Ne} / \mathrm{i}-\mathrm{C} 4)_{P}{ }^{\star}(\mathrm{i}-\mathrm{C} 4 / \mathrm{Ne})_{F}\right)$ as a function of the isobutane feed pressure at room temperature

When intracrystalline diffusion through the composite membrane is considered rate determining, the maximum flow can be calculated from equations (4) and (5), assuming Langmuir type adsorption. Some results are shown in Figure 5 for methane and $n$-butane at $20^{\circ} \mathrm{C}$ and applied low pressures. Self diffusion data for lower alkanes [11] were used to estimate the self diffusivity of $n$-butane. Sorption data on methane and n-butane were taken from [15] and [16], respectively. It can be seen that permeation is strongly depending on adsorption. For methane (weakly adsorbing) the molar flow is proportional with the applied feed pressure, as the driving force also increases. For stronger adsorbing molecules the maximum concentration on feed side is already reached at low pressures. According to the Fickian diffusional model a further pressure increase on feed side will not lead to higher flows, because the concentration profile over the zeolite film remains constant. The $n$-butane flow is however strongly depending on the permeate pressure, as the concentration difference over the membrane decreases.

The observed permeation rates (for permeate pressures $<100 \mathrm{~Pa}$ ) are two to three orders of magnitude lower than the calculated flows in Figure 5. Still, the flow is expected to be mainly governed by intracrystalline diffusion as has been shown by Barrer [5] for thick films (appr. $100 \mu \mathrm{m}$ ) and at room temperature. Apart from the extra resistance to mass flow the presence of the macroporous support on permeate side will also affect the permeation through the zeolite film. In the first place the effective surface area of the zeolite film is smaller than the total surface area, as a significant part of the film is directly bound to the non-permeable support particles. In addition, the flow through the support will be driven by a non-zero concentration gradient over the support, leading to a higher partial pressure on 
permeate side. Consequently, the driving force for permeation through the zeolite film will be reduced, although the effect will vary for each type of adsorbate as shown in Figure 5.

Also, the zeolite phase itself may lead to lower than ideal permeation rates, due to the at random orientation of the MFI type crystals. The diffusional anisotropy in MFI type zeolites has been demonstrated by the PFG NMR technique, even for small molecules like methane [17]. Very few crystals will be exactly oriented in the a- or b-direction (sinusoidal and straight channels), so permeation mainly proceeds via both channel types. Thus, an average diffusivity lower than for the straight or sinusoidal channels should be found. In fact the tortuosity is increased as well, because movement in the cdirection requires four times the number of steps per unit cell as compared to the $a$ - or b-direction. The possibility of lattice imperfections or channels that are partly or fully blocked by metal ions cannot be ruled out either.

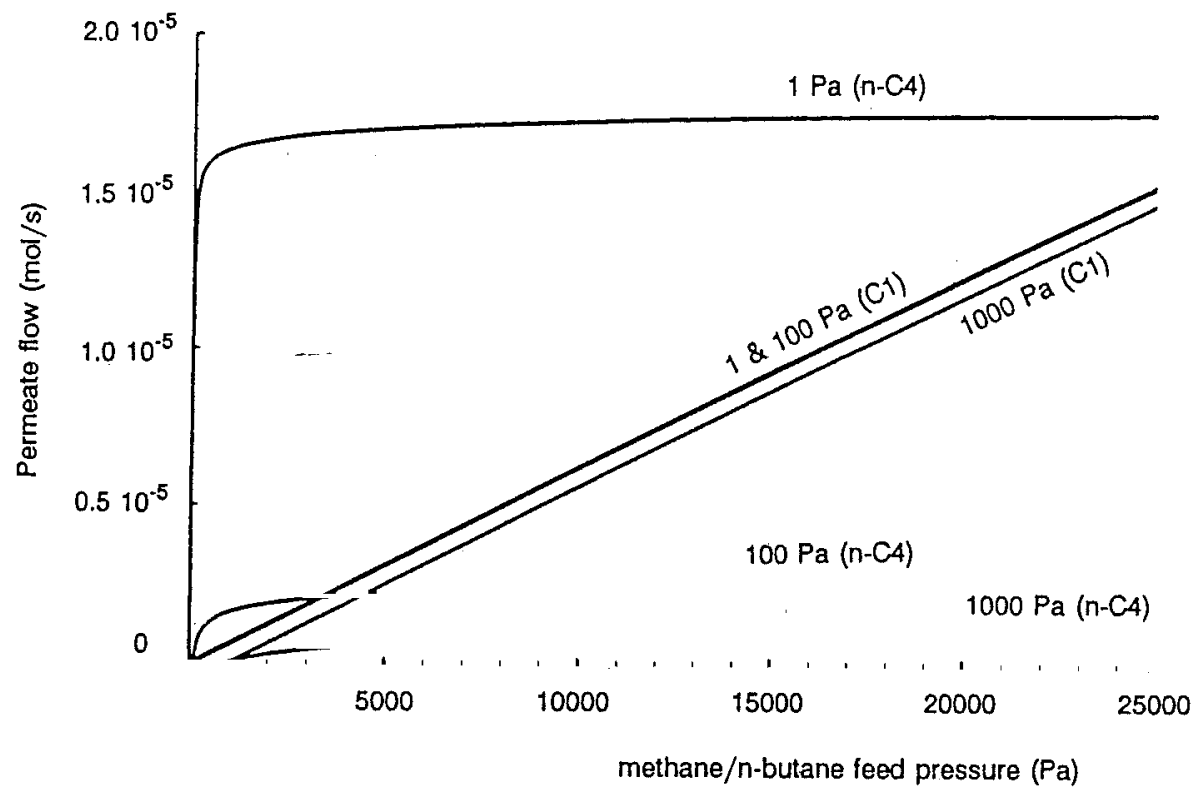

Figure 5. Theoretical flows for $n$-butane (n-C4) and methane (C1) as a function of the feed pressure and constant permeate pressure $\left(A_{m}=1.5 \mathrm{~cm}^{2} ; I_{m}=50 \mu \mathrm{m}\right)$

The limited data on adsorption do not allow for reliable calculation of permeation rates at high pressures. At feed and permeate pressures in excess of 1 bar permeation rates for $n$-butane are calculated to be in the order of $10^{-8} \mathrm{~mol} / \mathrm{s}$. Actually measured permeation rates on similar MFI/clay membranes, applying a pressure difference over the membrane (feed pressure 2 bar; permeate pressure $1 \mathrm{bar})$, were also in the order of $10^{-8} \mathrm{~mol} / \mathrm{s}$ [12]. The obvious conclusion would be that at sufficiently high pressures the intracrystalline diffusion is governing the permeation rate, as the resistance due to the support layer is negligible. It remains however unclear whether the here described diffusional model (Langmuir isotherm, Fickian diffusion) is appropriate to describe mass flow at such high (and industrially relevant) pressures. The effect of an additional driving force (pressure gradient) may be substantial.

\section{CONCLUSIONS}

Continuous films of the MFI type can be grown on a clay support. These in situ grown zeolite films have been characterized by membrane permeation experiments. Typical aspects of MFI type films, grwon with the aid of a template like TPA ${ }^{+}$were found to be: (i) gas-tightness for as-synthesized films, 
(ii) the occurrence of time lags due to the strong adsorption of sorbate molecules within the framework, (iii) multicomponent diffusion phenomena in transient state measurements (temporary maximum permeation rate for neon), (iv) shape selectivity for butane isomers. Another test would be to check the gas-tightness for too large a molecule to have access to the MFI micropores.

In order to describe the permeation at concentrations near saturation, it will be essential to study adsorption and diffusion processes outside of the Henry region. The experimental permeation rates are lower than calculated for exclusively intracrystalline diffusion. This seems to be at least partly due to the presence of the macroporous support, although the effect depends on the applied conditions on both feed and permeate side. Nevertheless the resistance to mass flow due to the macroporous support should be minimized, if only to establish the true maximum flow rates under relevant conditions. In addition thin zeolite films are required as the flow rate is expected to be inversely proportional to the thickness of the separating layer. Obviously, for most industrial applications large membrane surfaces will be needed, so the membrane preparation by in situ growth is favoured.

\section{ACKNOWLEDGEMENTS}

Financial support by the Technology Foundation (STW), Netherlands, project UCH 71.1334, is gratefully acknowledged.

\section{LITERATURE}

1. a) D.L. Wernick, E.J. Osterhuber, in: Proceedings of the 6th International Zeolite Conference, Eds. D.H. Olson, and A. Bisio, Reno, Nevada, July 10-15, (1983), 122-130; b) D.L. Wernick, E.J. Osterhuber, J.Membr.Sci. 22, (1985), 137-146.

2. a) A.R. Paravar, D.T. Hayhurst, in: Proceedings of the 6th International Zeolite Conference, Eds. D.H. Olson, and A. Bisio, Reno, Nevada, July 10-15, (1983), 217-224; b) D.T. Hayhurst, A.R. Paravar, Zeolites 8, (1988), 27-29.

3. E.R. Geus, A.E. Jansen, J.C. Jansen, J. Schoonman, H. van Bekkum, Proceedings of ZEOCAT 90, Eds. G. OhImann, H. Pfeifer, and R. Fricke, Stud.Surf.Sci.Catal. 65, Leipzig, Germany, August 20 23, (1990), 457-466.

4. W.O. Haag, J.G. Tsikoyiannis, US Patent $5,019,263$.

5. R.M. Barrer, in: Proceedings of ZEOCAT 90, Eds. G. Ohlmann, H. Pfeifer, and R. Fricke, Stud.Surf.Sci.Catal. 65, Leipzig, Germany, August 20-23, (1990), 257-273.

6. R.M. Barrer, J.Chem.Soc.Faraday Trans. 86, (1990), 1123-1130.

7. R.M. Barrer, Langmuir 3, (1987), 309-315.

8. J. Kärger, D.M. Ruthven, Zeolites 9, (1989), 267-281.

9. L.S. Darken, Trans. AIME 175, (1948), 184-194.

10. J. Kärger, S.P. Shdanov, A. Walter, Z.phys.Chemie 256, (1975), 319-329.

11. J. Caro, M. Bülow, W. Schirmer, J. Kärger, W. Heink, H. Pfeifer, S.P. Shdanov, J.Chem.Soc.Faraday Trans. 81, (1985), 2541-2550.

12. E.R. Geus, M.J. den Exter, H. van Bekkum, to be published.

13. E.R. Geus, M.J. den Exter, H. van Bekkum, Dutch Patent Application 91.01.148.

14. J. Kärger, H. Pfeifer, D. Freude, in: Proceedings of the 7th International Zeolite Conference, Eds. Y. Murakami, A. lijima, and J.W. Ward, Tokyo, Japan, August 17-22, (1986), 633-639.

15. L.V.C. Rees, Proceedings of ZEOCAT 90, Eds. G. Ohlmann, H. Pfeifer, and R. Fricke, Stud.Surf.Sci.Catal. 65, Leipzig, Germany, August 20-23, (1990),

16. R.E. Richards, L.V.C. Rees, Langmuir 3, 335-340.

17. U. Hong, J. Kärger, R. Kramer, H. Pfeifer, G. Seiffert, U. Müller, K.K. Unger, H.B. Lück, T. Ito, Zeolites 11, (1991), 816-821. 